\title{
NOTES ON THE INVERSION OF INTEGRALS II
}

\author{
GEORGE R. KEMPF
}

(Communicated by Jonathan M. Rosenberg)

\begin{abstract}
If $W$ is a Picard bundle on the Jacobian $J$ of a curve $C$, we have the problem of describing $W$ globally. The theta divisor $\theta$ is ample on $J$. Thus it is possible to write $W$ as the sheaf associated to a graded $M$ over the well-known ring $\oplus_{m \geq 0} \Gamma\left(J, \mathscr{O}_{J}\left(m^{4} \theta\right)\right)$. In this paper we compute the degree of generators and relations for such a module $M$.
\end{abstract}

In this part I will solve a problem which will allow the development of the normal presentation of twists of the Picard bundle on the Jacobian rather than their pull-back by isogenies. Also I will discuss the rigidity of Picard bundles pulled back by isogenies.

\section{THE RESTRICTION THEOREM}

Let $\mathscr{L}$ be an ample invertible sheaf on an abelian variety $X$ over $k=\bar{k}$. Let $1 \rightarrow \mathbb{G}_{m} \rightarrow H \stackrel{\alpha}{\rightarrow} K \rightarrow 0$ be Mumford's theta group of $\mathscr{L}$. Here $K$ is the closed subgroupscheme of $X$ given by $\operatorname{Ker}\left(\psi_{\mathscr{L}}\right)=K$ where $\psi_{\mathscr{L}}: X \rightarrow \widehat{X}$ is as usual. Take a maximal closed subgroupscheme $K_{1}$ of $K$ such that $\alpha^{-1}\left(K_{1}\right)$ is abelian. As the commutative extension of $K_{1}$ by $\mathbb{G}_{m}$ splits, we may choose a homomorphism $\sigma: K_{1} \rightarrow H$ such that $\alpha \circ \sigma=1_{K_{1}}$.

Let $x$ be a point of $X$. Then we have a restriction homomorphism $R(x)$ : $\Gamma(X, \mathscr{L}) \rightarrow \Gamma\left(x+K_{1},\left.\mathscr{L}\right|_{x+K_{1}}\right)$. Our first result is

Lemma 1. For $x$ in a non-empty open subset of $X$, the map $R(x)$ is an isomorphism.

Proof. By standard theory both spaces have the same dimension. Better yet they are both the regular representation of $K_{1}$. Let's see how the representations occur. By definition we have a given $H$-linearization of $\mathscr{L}$ and, hence, an induced $\alpha^{-1}\left(K_{1}\right)$-linearization of $\left.\mathscr{L}\right|_{x+K_{1}}$. The restriction $R(x)$ is obviously a $\alpha^{-1}\left(K_{1}\right)$-homomorphism. Thus via $\alpha$ the restriction $R(x)$ is a homomorphism of $K_{1}$-modules. by [7 or 8] $\Gamma(X, \mathscr{L})$ is isomorphic to the regular representation of $K_{1}$. The same holds for $\Gamma\left(x+K_{1},\left.\mathscr{L}\right|_{x+K_{1}}\right)$ as $\left.\mathscr{L}\right|_{x+K_{1}}$ is a $K_{1}$-linearized

Received by the editors February 2, 1989, and in revised form April 10, 1989.

1980 Mathematics Subject Classification (1985 Revision). Primary 14H40; Secondary 14K30.

Key words and phrases. Algebraic curves, Jacobians and Picard bundles. 
invertible sheaf on a principal homogeneous space of $K_{1}$ (in other words $\Gamma(x+$ $\left.K_{1},\left.\mathscr{L}\right|_{x+K_{1}}\right)$ is induced from the trivial one-dimensional representation of the identity subgroup of $K_{1}$ ).

To check that $R(x)$ is an isomorphism we will use the criterion

(1) $R(x)\left(t_{\chi}\right) \neq 0$ for all eigenvectors $t_{\chi}$ with eigenvalue a character $\chi$ of $K_{1}$.

This criterion is clear because any non-zero $K_{1}$-submodule $\operatorname{Ker}(R(x))$ of $\Gamma(X, \mathscr{L})$ contains an eigenvector because $K_{1}$ is abelian. The next point is that up to constant multiple $t_{\chi}$ is determined by $\chi$ because $\Gamma(X, \mathscr{L})$ is the regular representation. Therefore we need only check a finite number of conditions for our criterion. As $t_{\chi} \neq 0$ it has non-zero value at most points $x$ of $X$. Therefore $R(x)\left(t_{\chi}\right) \neq 0$ for most $x$ and all of the finitely many $\chi$.

Now let $\mathscr{M}$ be another ample invertible sheaf on $X$. Then we have a restriction

$$
S(x): \Gamma(X, \mathscr{L} \otimes \mathscr{M}) \rightarrow \Gamma\left(X+K_{1},\left.\mathscr{L} \otimes \mathscr{M}\right|_{x+K_{1}}\right),
$$

which satisfies

Theorem 2. For all points $x$ of $X$ the restriction $S(x)$ is surjective.

Remark. When $\mathscr{M}=\mathscr{L}$ the result implies that $\Gamma\left(X, \mathscr{L}^{\otimes 2}\right)$ generates $\mathscr{L}^{\otimes 2}$ which is well-known.

Remark. In some classical cases Theorem 2 is due to S. Koizumi and called by him the "rank theorem" $[5,6]$.

Proof. Let $\theta$ be the zeroes of a section $\sigma$ of $\mathscr{M}$. Then for fixed $x$ $(\theta+y) \cap\left(x+K_{1}\right)=\varnothing$ if and only if $y \in-\theta+x+K$. Thus for general $y,\left.T_{y}^{*} \sigma\right|_{x+K_{1}}$ is nowhere vanishing section of $\left.T_{y}^{*} \mathscr{M}\right|_{x+K_{1}}$, where $T_{y}: X \rightarrow X$ is translation by $y$. Now $T_{y}^{*} \mathscr{M}$ runs through all sheaves algebraically equivalent to $\mathscr{M}$ as $\mathscr{M}$ is ample.

Thus we may find $\mathscr{M}^{\prime}$ and $\mathscr{L}^{\prime}$ algebraically equivalent to $\mathscr{M}$ and $\mathscr{L}$ such that $\mathscr{M}^{\prime} \otimes \mathscr{L}^{\prime} \approx \mathscr{M} \otimes \mathscr{L}$ so that the restriction $S(x): \Gamma(X, \mathscr{M} \otimes \mathscr{L}) \rightarrow \Gamma(x+$ $\left.\left.K_{1}, \mathscr{M} \otimes \mathscr{L}\right)\left.\right|_{x+K_{1}}\right)$ is surjective if the restriction $R^{\prime}(x): \Gamma\left(X, \mathscr{L}^{\prime}\right) \rightarrow \Gamma(x+$ $\left.K_{1},\left.\mathscr{L}^{\prime}\right|_{x+K_{1}}\right)$ is surjective where $\mathscr{L}^{\prime}$ is general of its type. This follows by multiplying a section of $\Gamma\left(X, \mathscr{M}^{\prime}\right)$ which vanishes nowhere on $x+K_{1}$.

I claim the previous lemma means that $R^{\prime}(x)$ is an isomorphism for general $\mathscr{L}^{\prime}$. This claim implies the theorem from the above. For the claim take $\mathscr{L}^{\prime}=$ $T_{y}^{*} \mathscr{L}$. Then $R^{\prime}(x) \approx R(x-y)$. Hence the claim follows from the lemma.

\section{Global SPANNING}

Let $\mathscr{V}(\mathscr{L})=\pi_{\widehat{X}^{*}}\left(\pi_{X}^{*}(\mathscr{L}) \otimes \mathscr{P}\right)$ where $\mathscr{P}$ is a Poincaré sheaf on $X \times \widehat{X}$ where $\widehat{X}$ is the dual abelian variety. Let $\mathscr{N}$ be an invertible sheaf on $\widehat{X}$. We want to know when $\mathscr{V}(\mathscr{L}) \otimes \mathscr{N}$ is spanned by its global sections. 
Let $Y$ be $X / K_{1}$. As $K_{1} \subset \operatorname{Ker}\left(\psi_{\mathscr{L}}\right)$ we have a factorization $X \stackrel{\alpha}{\rightarrow} Y \stackrel{b}{\rightarrow} \widehat{X}$ of $\psi_{\mathscr{L}}$. Let $L$ be the closed subgroupscheme $K / K_{1}$ of $Y$. Let $\mathscr{Q}$ be the invertible sheaf on $y$ gotten by descending $\mathscr{L}$ with the $K_{1}$-action given by the homomorphism $\sigma$. We will assume that $\operatorname{char}(k) \nmid \operatorname{deg}\left(\psi_{\mathscr{L}}\right)$.

Lemma 3. $\mathscr{V}(\mathscr{L}) \otimes \mathscr{N}$ is spanned by its global sections if and only if the restriction

$$
U(y): \Gamma\left(Y, \mathscr{Q}^{\otimes-1} \otimes b^{*} \mathcal{N}\right) \rightarrow \Gamma\left(y+L,\left.\mathscr{Q}^{\otimes-1} \otimes b^{*} \mathcal{N}\right|_{y+L}\right)
$$

is surjective for all points $y$ of $Y$.

Proof. For $y$ in $Y, \mathscr{V}(\mathscr{L}) \otimes \mathscr{N}$ is spanned by its sections at $b(y)$ if and only if the restriction $\Gamma(X, \mathscr{V}(\mathscr{L}) \otimes \mathscr{N}) \rightarrow \mathscr{V}(\mathscr{L}) \otimes \mathscr{N}(b(y))$ is surjective if and only if the pull-back plus restriction $W(y): \Gamma(X, \mathscr{V}(\mathscr{L}) \otimes \mathscr{N}) \rightarrow\left(b^{*}(\mathscr{V}(\mathscr{L}) \otimes \mathscr{N})(y)\right.$ is surjective. As $b$ is surjective, $\mathscr{V}(\mathscr{L}) \otimes \mathscr{N}$ is spanned by its global sections if and only if $W(y)$ is surjective for all $y$ in $Y$. Thus it will be enough to prove that $W(y)$ is surjective if and only if $U(y)$ is surjective. To show this I intend to give a commutative diagram

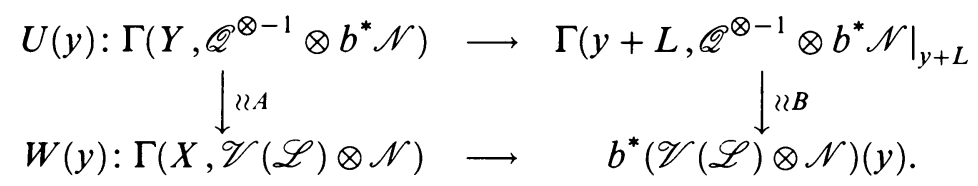

We need to compute the sheaf $b^{*}(\mathscr{V}(\mathscr{L}) \otimes \mathscr{N})$ together with its $L$-linearization. Let $\chi$ be a character of $K_{1}$. We have a sheaf $\mathscr{Q}_{\chi}$ on $Y$ gotten by descending the action of $K_{1}$ on $\mathscr{L}$ via $\sigma$ twisted by $\chi$. Thus $\mathscr{Q}_{1}=\mathscr{Q}$ and all of the $\mathscr{Q}$ 's are algebraically equivalent. The first step is

Sublemma 4. We have a natural isomorphism

$$
b^{*}(\mathscr{V}(\mathscr{L}) \otimes \mathscr{N}) \simeq \bigoplus_{\chi \in K_{1}} \Gamma\left(Y, \mathscr{Q}_{\chi}\right) \otimes_{k}\left(\mathscr{Q}_{\chi}^{\otimes-1} \otimes b^{*} \mathscr{N}\right),
$$

where the spaces $\Gamma\left(Y, \mathscr{Q}_{\chi}\right)$ are lines.

Proof. As the $K_{1}$-module $\Gamma(X, \mathscr{L})$ is the regular representation, it is the direct sum of its $\chi$-eigenspaces $\Gamma(\chi, \mathscr{L})^{\chi}$ which are lines. On the other hand $\Gamma(X, \mathscr{L})^{\chi}=\Gamma\left(X / K_{1}, \mathscr{L}^{\chi}\right)=\Gamma\left(Y, \mathscr{Q}_{\chi}\right)$. Thus the spaces are lines.

Now by [1] we have a canonical isomorphism $\left(\psi_{\mathscr{L}}\right)^{*} \mathscr{V}(\mathscr{L}) \approx \Gamma(X, \mathscr{L}) \otimes_{k}$ $\mathscr{L}^{\otimes-1}$ where the $K_{1}$-action (even $K$-action) is the obvious one. As $\psi_{\mathscr{L}}=$ $b \circ a, b^{*} \mathscr{V}(\mathscr{L})$ is the sheaf of $K_{1}$-invariants in $\Gamma(X, \mathscr{L}) \otimes_{k} \mathscr{L}^{\otimes-1}$ which is $\oplus_{\chi} \Gamma(X, \mathscr{L})^{\chi} \otimes_{k}\left(\mathscr{L}^{\otimes-1}\right)^{\chi-1}=\oplus_{\chi} \Gamma(X, \mathscr{L})^{\chi} \otimes_{k}\left(\mathscr{L}^{\chi}\right)^{\otimes-1}=\oplus \Gamma\left(Y, \mathscr{Q}_{\chi}\right) \otimes_{k} \mathscr{Q}_{\chi}^{\otimes-1}$. The sublemma results by tensoring this isomorphism with $b^{*} \mathscr{N}$.

The second step gives rise to $L$-action under this isomorphism. For simplicity of exposition we will assume that there is another maximal subgroup $K_{2}$ of $K$ with a section $\tau$ of $\alpha$ over $K_{2}$ such that $K_{2} \cap K_{1}=\{0\}$. By projection $K_{2} \approx L$ and via the Weil form $e_{\mathscr{L}}$ of $\mathscr{L}, K_{2}$ (and hence $L$ ) may be identified with 
$\widehat{K}_{1}$. Let $\psi(e)$ denote the character of $K_{1}$ corresponding to an element $\ell$ of $L$. The crucial fact is that

$\left(^{*}\right)$ for any $\chi$ in $K_{1}$ and $\ell$ in $L$ we have a $T_{e}$-isomorphism $\rho(\ell, \chi): \mathscr{Q}_{\chi} \rightarrow \mathscr{Q}_{\chi \psi(\ell)}$ such that $\rho\left(\ell_{2}, \chi \psi\left(\ell_{1}\right)\right) \circ \rho\left(\ell_{1}, \chi\right)=\rho\left(\ell_{1}+\ell_{2}, \chi\right)$.

Here The $T_{k}$-isomorphism $a^{*}(\rho, \chi): \mathscr{L} \rightarrow \mathscr{L}$ is just the action of the element $k$ of $K$ over $\ell$ on $\mathscr{L}$ via $\tau$. The above fact results from the study of how the $K_{2}$-action on $\mathscr{L}$ falls to commute with that of $K_{1}$. Thus we have isomorphism

$$
\Gamma(y, \rho(\ell, \chi)): \Gamma\left(Y, \mathscr{Q}_{\chi}\right) \stackrel{\approx}{\rightarrow} \Gamma\left(Y, \mathscr{Q}_{\chi \psi(\ell)}\right)
$$

and the $T_{e}$-isomorphism

$$
\left(\rho(-\ell, \chi \psi(\ell)) \otimes K_{e}: \mathscr{Q}_{\chi}^{\otimes-1} \otimes b^{*} \mathcal{N} \rightarrow \mathscr{Q}_{\chi \psi(\ell)}^{\otimes-1} \otimes b^{*} \mathcal{N},\right.
$$

where $K$, is the action of $\ell$ on $b^{*} \mathcal{N}$. Summing up without any more details we get

Sublemma 5. The L-action on $\bigoplus_{\chi} \Gamma\left(y, \mathscr{Q}_{\chi}\right) \otimes_{k}\left(\mathscr{Q}_{\chi}^{\otimes-1} \otimes b^{*} \mathscr{N}\right)$ is the direct sum of the tensor products of the above isomorphisms.

Now $\Gamma(\widehat{X}, \mathscr{V}(\mathscr{L}) \otimes \mathscr{N})$ is the space of $L$-invariants in $\Gamma\left(Y, b^{*}(\mathscr{V}(\mathscr{L}) \otimes\right.$ $\mathscr{N}))$. Thus

$$
\Gamma(\widehat{X}, \mathscr{V}(\mathscr{L}) \otimes \mathscr{N})=\left(\bigoplus_{\chi} \Gamma\left(Y, \mathscr{Q}_{\chi}\right) \otimes_{k} \Gamma\left(Y, \mathscr{Q}_{\chi}^{\otimes-1} \otimes b^{*} \mathscr{N}\right)\right)^{L} .
$$

Explicitly all such $L$-invariants are

$$
M(a)=\sum_{\ell} \Gamma(Y, \rho(\ell, 1)) \cdot d \times \Gamma\left(\rho(-\ell, \psi(\ell))^{\otimes-1} \otimes K_{e}\right) a
$$

where $\ell$ is a fixed non-zero element of $\Gamma(y, \mathscr{Q})$ and $a$ is an arbitrary element of $\Gamma\left(Y, \mathscr{Q}^{\otimes-1} \otimes b^{*} \mathscr{N}\right)$. The isomorphism $A$ is just the correspondence between invariants and $a$ 's.

Next we need to evaluate the section $M(a)$ at a point $y$ of $Y$. The value of $M(a)$ at $y$ is an element of $\bigoplus, \Gamma\left(Y, \mathscr{Q}_{\psi(\zeta)}\right) \otimes_{k}\left(\mathscr{Q}_{\psi(\zeta)}^{\otimes-1} \otimes b^{*} \mathscr{N}\right)(y) \approx$ $\bigoplus, \Gamma(Y, \mathscr{Q}) \otimes_{k}\left(\mathscr{Q}^{\otimes-1} \otimes b^{*} \mathcal{N}\right)(y+\ell)=\Gamma(Y, \mathscr{Q}) \otimes \Gamma\left(y+L,\left.\mathscr{Q}^{\otimes-1} \otimes b^{*} \mathcal{N}\right|_{y+L}\right)$ under the isomorphism $M(a)(y)$ goes to $1 \otimes \sum_{\ell \in L} a(y+\ell) \delta_{\ell}$. Using these isomorphisms we have the isomorphism $B$ and the required commutative diagram.

Now we are ready to put together the previous results. Let $\mathscr{R}$ be an ample invertible sheaf on $X$ such that $\psi_{\mathscr{R}}: X \rightarrow \widehat{X}$ is an isomorphism which we will take to be an identification. Thus $X$ is principally polarized. Assume that $\psi_{\mathscr{L}}=\ell 1_{X}$ and $\psi_{\nu}=\mathscr{N} 1_{X}$. Then we have $\ell$ and $n>0$ as $\mathscr{N}$ and $\mathscr{L}$ are ample. 
Theorem 6. If $\ell(n-1)>1$ then $\mathscr{V}(\mathscr{L}) \otimes \mathscr{N}$ is generated by its sections whenever char $(k)+\ell$.

Proof. Choose a decomposition $K_{1} \oplus K_{2}$ of $X_{\ell}=\operatorname{Ker}\left(\ell 1_{X}\right)$ by subgroups which are isotropic with respect to the Weil form of $\mathscr{L}$. Let $Y=X / K_{1}$. Then $Y$ is principally polarized by $\mathscr{Q}$ where $a^{*} \mathscr{Q} \approx \mathscr{L}$ and $X \stackrel{a}{\rightarrow} Y \stackrel{b}{\rightarrow} X$ is the factorization of $\ell 1_{X}$. The classifying homomorphism $\psi_{b^{*} \mathscr{N}}: Y \rightarrow Y$ is $n \ell 1_{Y}$ by an elementary calculation. Thus $\mathscr{Q}^{\otimes-1} \otimes b^{*} \mathcal{N}$ is algebraically equivalent to $\mathscr{Q}^{n /-1}$. By Lemma 3 we need to check whether $\Gamma\left(Y, \mathscr{Q}^{\otimes-1} \otimes b^{*} \mathcal{N}\right) \rightarrow \Gamma(y+$ $\left.L,\left.\mathscr{Q}^{\otimes-1} \otimes b^{*} \mathcal{N}\right|_{y+L}\right)$ is surjective for all $y$ in $Y$ where $L$ is the isomorphic image of $K_{2}$ in $Y$.

Now $L$ is a maximal isotopic subgroup of $Y_{\ell}=\operatorname{Ker}\left(\psi_{\mathscr{Q}} \otimes \ell\right)$ with respect to $e_{\mathscr{\ell}} \otimes \ell$. If $(n-1) \ell>1,\left(\mathscr{Q}^{\otimes-\ell} \otimes b^{*} \mathscr{N} \otimes \mathscr{Q}^{\otimes-1}\right) \equiv \mathscr{M}$ is ample. Thus the restriction is surjective by Theorem 2 .

\section{Normal presentation for Picard bundles}

We will be using the notation of Part I [4].

Theorem 7. (a) $\mathscr{U}_{n}(D) \otimes \mathscr{M}$ is normally presented for $\mathscr{R}$ if $m \geq 3$ and $r \geq 4$. If furthermore char $(k)+m$ then

(b) it is strongly normally presented and

(c) the multiplication $\Gamma\left(J, \mathscr{U}_{n}(D) \otimes \mathscr{M}\right) \otimes \Gamma(J, \mathscr{R}) \rightarrow \Gamma\left(J, \mathscr{U}_{n}(D) \otimes M \otimes \mathscr{R}\right)$ is surjective.

Proof. The first point is that $\mathscr{U}_{n}(D)$ only depends on $\left.\mathscr{L}_{n}\right|_{C}(-D)$. So choosing $\mathscr{L}_{n}$ and $D$ correctly we may assume that $\operatorname{char}(k)+n$ and $D$ is reduced. So by Theorem $6 \mathscr{V}_{n} \otimes \mathscr{M}$ is generated by its sections. Then we proceed as in the proof of Part I, Theorem 7. As $\mathscr{V}_{n} \rightarrow \mathscr{W}_{n g}$ is surjective, $\mathscr{W}_{n g} \otimes \mathscr{M}$ is generated by its sections. From the exact sequence

$$
0 \rightarrow \mathscr{W}_{n g} \otimes \mathscr{M} \rightarrow \bigoplus_{1 \leq i \leq d} \mathscr{S}_{i} \otimes \mathscr{M} \rightarrow \mathscr{U}_{n}(D) \otimes \mathscr{M} \rightarrow 0
$$

and Part I, Lemma 2 we need only see for (a) $\mathscr{S}_{i} \otimes \mathscr{M}$ is strongly normally presented and for (b) $H^{1}\left(J, \mathscr{W}_{n g} \otimes \mathscr{M}\right)=0$. The first statement is Part I, Theorem 6 and the second follows from [1, Theorem 3.8] when $n>2$, which we may assume. This proves (a) and (b).

For (c) by the above it suffices to show that the multiplier is surjective

$$
\Gamma\left(J, \mathscr{S}_{i} \otimes \mathscr{M}\right) \otimes \Gamma(J, \mathscr{R}) \rightarrow \Gamma\left(J, \mathscr{S}_{i} \otimes \mathscr{M} \otimes \mathscr{R}\right)
$$

but this follows from the Mumford-Koizumi Theorem [3].

Next we compute the dimension of sections of twists of $\mathscr{U}_{n}(D)$.

Theorem 8. (a) If $m>0$ then $H^{i}\left(J, \mathscr{U}_{n}(D) \otimes \mathscr{M}\right)=0$ if $i>0$,

(b) $\Gamma\left(J, \mathscr{U}_{n}(D) \otimes \mathscr{M}\right)=H^{1}\left(C,\left.\mathscr{L}_{n}\right|_{C}(-D) \otimes \pi_{C^{*}}\left(\left.\pi_{2}^{*} \mathscr{M} \otimes \mathscr{P}\right|_{C \times J}\right)\right)$, and

(c) $\operatorname{dim} \Gamma\left(J, \mathscr{U}_{n}(D) \otimes \mathscr{M}\right)=(d-g n+g-1) m^{g}+g m^{g-1}$. 
Proof. By (a) the dimension equals the Euler characteristic of $\mathscr{U}_{n}(D) \otimes \mathscr{M}$, which by the Riemann-Roch Theorem is the number of points in $\operatorname{ch}\left(\mathscr{U}_{n}(D) \otimes \mathscr{M}\right)$ which equals $($ rank $+\theta) \exp (m \theta)$. (See Theorem 8 in Part I). Thus (c) follows.

For (a) and (b) as $\mathscr{U}_{n}(D)=R_{\pi_{J^{*}}}^{1}\left(\left.\pi_{1}^{*} \mathscr{L}_{n} \otimes \mathscr{P}\right|_{C \times J}(-D \times J)\right)$ and the other direct images are zero, we have an isomorphism $H^{i}\left(J, \mathscr{U}_{n}(D) \otimes \mathscr{M}\right) \approx H^{i+1}(C \times$ $\left.J,\left.\pi_{1}^{*} \mathscr{L}_{n} \otimes \mathscr{P} \otimes \pi_{2}^{*} \mathscr{M}\right|_{C \times J}(-D \times J)\right)$ but as $m>0$ the higher direct images of the last sheaf via $\pi_{C}$ are zero [1]. Therefore

$$
\begin{aligned}
& H^{i+1}\left(C \times J,\left.\pi_{1}^{*} \mathscr{L}_{n} \otimes \mathscr{P} \otimes \pi_{2}^{*} \mathscr{M}\right|_{C \times J}(-D \times J)\right) \\
& =H^{i+1}\left(C,\left.\left.\mathscr{L}_{n}\right|_{C}(-D) \otimes \pi_{1^{*}}\left(\mathscr{P} \otimes \pi_{2}^{*} \mathscr{M}\right)\right|_{C}\right) .
\end{aligned}
$$

As $C$ is a curve the last cohomology group is zero if $i>0$. Thus (a) and (b) follow from the two isomorphisms.

\section{RIgIDITY OF THE PICARD UNDER PULL-BACKS}

As is well-known the Picard bundles $\mathscr{U}_{n}(D)$ describe the fibering $\int: C^{(r)} \rightarrow J$ of the symmetric product $C^{(r)}$ over the Jacobian $J$ for $r>2 g-2$. The rigidity of $\mathscr{U}_{n}(D)$ translates into a statement about the deformations of $C^{(r)}$ [2]. In the current situation we want to study the deformations of a variety $X$ where $X=C^{(r)} \times{ }_{J} A$ for an isogeny $f: A \rightarrow J$ of degree prime to the characteristic. Thus $X$ is an abelian unramified covering of $C^{(r)}$ of degree prime to $\operatorname{char}(k)$.

Theorem 9. If $r>3$ and $C$ has general moduli then any deformation of $X$ is induced by a deformation of $C$ and a deformation of the isogeny $f$.

We will prove this theorem later. Let $\operatorname{Pic}^{0}(Y)$ be the connected component of the Picard scheme of a variety $Y$. We will begin by proving

Theorem 10. The natural mapping $\operatorname{Pic}^{0}\left(C^{(r)}\right) \rightarrow \operatorname{Pic}^{0}(X)$ is an isogeny of abelian varieties if $r>1$.

Proof. First recall that $\mathrm{Pic}^{0}\left(C^{(r)}\right)$ is isomorphic to the Jacobian $J$. A key fact is that for any two morphisms $g$ and $h: S \rightarrow J,(g+f)^{*}: \operatorname{Pic}^{0}(J) \rightarrow \operatorname{Pic}^{0}(S)$ is the product $g^{*} \otimes f^{*}$ (this is the theorem of the square). To use this fact look at $\operatorname{Pic}^{0}(J) \rightarrow \operatorname{Pic}\left(C^{(r)}\right)^{i} \rightarrow \operatorname{Pic}^{0}\left(C^{\times r}\right)$. Then the composition sends $[\mathscr{L}]$ to $\left[\bigotimes_{i} \pi_{i}^{*}\left(\left.\mathscr{L}\right|_{C}\right)\right]$. By autoduality of the Jacobian $\operatorname{Pic}^{0}(J) \approx \operatorname{Pic}^{0}(C) \approx J$. Thus the composition is $J \rightarrow \operatorname{Pic}^{*}\left(C^{(r)}\right) \rightarrow J$. By the fixed point argument [2, Lemma 1.3] with $G=\mathbb{G}_{m}$ pull-back gives an inclusion $H^{1}\left(C^{(r)}, \mathscr{O}_{C^{(r)}}^{*}\right) \hookrightarrow$ $\left(H^{1}\left(C^{\times r}, \mathscr{O}_{C \times r}^{*}\right)^{\operatorname{Sym}(r)}\right.$. Thus $i$ is an inclusion and hence an isomorphism with $J=\left(J^{\times r}\right)^{\operatorname{Sym}(r)}$ (set-theoretically). To remove this qualification we use the argument when $G=\mathbb{G}_{a}$. Hence $H^{1}\left(C^{(r)}, \mathscr{O}_{C^{(r)}}\right) \hookrightarrow\left(H^{1}\left(C^{\times r}, \mathscr{O}_{C \times r}\right)^{\operatorname{Sym}(r)}\right.$ is injective and, hence, $\operatorname{Pic}^{0}\left(C^{(r)}\right) \approx J$ because we have an isomorphism on tangent spaces. 
We next apply the fixed point argument to the covering $Y=C^{\times r} \times_{C^{(r)}} X \rightarrow$ $X$ of $X$ with Galois group $\operatorname{Sym}(r)$. Then we get an injection $\operatorname{Pic}^{0}(X) \hookrightarrow$ $\operatorname{Pic}^{0}(Y)^{\operatorname{Sym}(r)}$. We need to see that the composition $H^{1}\left(C^{(r)}, \mathscr{O}_{C^{(r)}}\right) \rightarrow$ $H^{1}\left(X, \mathscr{O}_{X}\right) \hookrightarrow H^{1}\left(Y, \mathscr{O}_{Y}\right)^{\operatorname{Sym}(r)}$ is an isomorphism, or, rather, $H^{1}\left(C^{\times r}, \mathscr{O}_{C \times r}\right)^{\mathrm{Sym}(r)} \rightarrow H^{1}\left(Y, \mathscr{O}_{Y}\right)^{\mathrm{Sym}(r)}$ is an isomorphism. Better yet we have Claim. $H^{1}\left(C^{\times r}, \mathscr{O}_{C \times r}\right) \rightarrow H^{1}\left(Y, \mathscr{O}_{Y}\right)$ is an isomorphism.

This uses Kummer theory. Let $K$ be the kernel of $f: A \rightarrow J$. Then for each character $\chi$ of $K$ we have an invertible sheaf $\mathscr{O}_{J}(\chi)$ on $J$ gotten by descending the translation action of $K$ on $\mathscr{O}_{K}$ by $\chi$. Then $\mathscr{O}_{J}(\chi)$ is contained in $\operatorname{Pic}^{0}(J)$. For any morphism $k: S \rightarrow J$ we define $\mathscr{O}_{S}(\chi)$ to be $k^{*} \mathscr{O}_{J}(\chi)$. Then by the above key fact $\mathscr{O}_{C \times r}(\chi)=\bigotimes_{i} \pi_{i}^{*} \mathscr{O}_{C}(\chi)$. Hence by the Künneth formula, if $\chi \neq 1, H^{1}\left(C^{\times r} \mathscr{O}_{C \times r}(\chi)\right)=0$ as $r>1$ and $H^{0}\left(C, \mathscr{O}_{C}(\chi)\right)=0$ because $\mathscr{O}_{C}(\chi)$ has degree zero and is not trivial. Now $\alpha^{*} \mathscr{O}_{Y}=\bigoplus_{\chi} \mathscr{O}_{C^{\times r}}(\chi)$ where $\alpha: Y \rightarrow C^{\times r}$ is the projection. Thus $H^{1}\left(Y, \mathscr{O}_{Y}\right)=\bigoplus_{\chi}\left(H^{1}\left(C^{\times r}, \mathscr{O}_{C \times r}(\chi)\right)=H^{1}\left(C^{\times r}, \mathscr{O}_{C \times r}\right)\right.$. This proves the claim.

I checked that the mapping of Theorem 10 is just $f^{\wedge}: J^{\wedge} \rightarrow A^{\wedge}$.

What we will need is the same fact for $\alpha^{\prime}: X \rightarrow C^{(r)}$.

Corollary 11. If $\chi \neq 1$, then $H^{1}\left(C^{(r)}, \mathscr{O}_{C^{(r)}}(\chi)\right)=0$ if $r>1$ and $H^{0}\left(C^{(r)}\right.$, $\left.\mathscr{O}_{C^{(r)}}(\chi)\right)=0$ if $r \geq 1$.

Proof. The second fact is a consequence of $\mathscr{O}_{C(r)}(\chi)$ being non-trivial but numerically trivial.

Now we can start the

Proof of Theorem 9. We need to compute $H^{1}\left(X, \theta_{X}\right)$. Now $\sigma_{*} \theta_{X} \approx \theta_{C^{(r)}} \otimes$ $\sigma_{*} \mathscr{O}_{X}=\theta_{C^{(r)}} \otimes \bigoplus_{\chi} \mathscr{O}_{C^{(r)}}(\chi)$. Thus we have a decomposition $H^{1}\left(X, \theta_{X}\right)=$ $\bigoplus_{\chi} H^{1}\left(C^{(r)}, \theta_{C^{(r)}} \otimes \mathscr{O}_{C^{(r)}}(\chi)\right)$. We need to see that

$$
\text { if } \chi \neq 1 \text { then } H^{1}\left(C^{(r)}, \theta_{C^{(r)}} \otimes \mathscr{O}_{C^{(r)}}(\chi)\right)=0
$$

because the theorem will follow from $A$ by deformation theory.

We will use the method of [2]. Let $D_{r}$ be the universal divisor on $C \times C^{(r)}$. Then $\theta_{C^{(r)}}=\tau_{*}\left(\left.\mathscr{O}_{C \times C^{(r)}}\left(D_{r}\right)\right|_{D_{r}}\right)$ where $\tau_{v}: D_{r} \rightarrow C^{(r)}$ is the projection. Thus $H^{i}\left(C^{(r)}, \theta_{C^{(r)}} \otimes \mathscr{O}_{C^{(r)}}(\chi)\right) \simeq H^{i}\left(D_{r}, \mathscr{O}_{C \times C^{(r)}}\left(\left.D_{r}\right|_{D_{r}} \otimes \tau_{r}^{*} \mathscr{O}_{C^{(r)}}(\chi)\right)\right.$. The first point is

Claim. If $r>2$ and $n \geq 0$ then

$$
\begin{aligned}
& H^{1}\left(D_{r}, \pi_{C}^{*}\left(\left.\left(\theta_{C} \otimes \mathscr{O}_{C}(\chi)^{\otimes n}\right)\left(D_{r}\right)\right|_{D_{r}} \otimes \tau_{r}^{*} \mathscr{O}_{C^{(r)}}(\chi)\right)\right. \\
& \quad \approx \quad \rightarrow H^{1}\left(D_{r-1}, \pi_{C}^{*}\left(\left.\left(\theta_{C} \otimes \mathscr{O}_{C}(\chi)^{\otimes n+1}\right)\left(D_{r-1}\right)\right|_{D_{r-1}} \otimes \tau_{v-1}^{*} \mathscr{O}_{C^{(r-1)}}(\chi)\right) .\right.
\end{aligned}
$$


Proof of claim. We have an isomorphism

$$
\alpha_{r}: C \times C^{(r-1)} \stackrel{\approx}{\rightarrow} D_{r}
$$

where

$$
\alpha_{r}^{*}\left(\left.\mathscr{O}_{C \times C^{(r)}}\left(D_{r}\right)\right|_{D_{r}}\right) \simeq \pi_{C}^{*} \theta_{C}\left(D_{r-1}\right)
$$

and

$$
\alpha_{r}^{*} \tau_{r}^{*} \mathscr{O}_{C^{(r)}}(\chi)=\pi_{C}^{*} \mathscr{O}_{C}(\chi) \otimes \pi_{C^{(r-1)}}^{*} \mathscr{O}_{C^{(r-1)}}(\chi) .
$$

Thus

$$
\left.\pi_{C}^{*}\left(\left(\theta_{C} \otimes \mathscr{O}_{C}(\chi)\right)^{\otimes n}\right)\left(D_{r}\right)\right|_{D_{r}} \otimes \tau_{r}^{*} \mathscr{O}_{C^{(r)}}(\chi)
$$

corresponds via $\alpha_{r}$ to the sheaf

$$
\left(\pi_{C}^{*}\left(\left(\theta_{C} \otimes \mathscr{O}_{C}(\chi)\right)^{\otimes n+1}\right) \otimes \pi_{C^{(r-1)}}^{*} \mathscr{O}_{C^{(r-1)}}(\chi)\right)\left(D_{r-1}\right) .
$$

Using the sequence

$$
\left.0 \rightarrow \mathscr{O}_{C \times C^{(-1)}} \rightarrow \mathscr{O}_{C \times C^{(r-1)}}\left(D_{r-1}\right) \rightarrow \mathscr{O}_{C \times C^{(r-1)}}\left(D_{r-1}\right)\right|_{D_{r-1}} \rightarrow 0
$$

tensored by

$$
\left(\pi _ { C } ^ { * } \left(\left(\theta_{C} \otimes \mathscr{O}_{C}(\chi)^{\otimes n+1}\right) \otimes \pi_{C^{(r-1)}}^{*} \mathscr{O}_{C^{(r-1)}}(\chi),\right.\right.
$$

we see that

$$
\begin{aligned}
& H^{1}\left(C \times C^{(r-1)},\left(\pi_{C}^{*}\left(\left(\theta_{C} \otimes \mathscr{O}_{C}(\chi)^{\otimes n+1}\right) \otimes \pi_{C^{(r-1)}}^{*} \mathscr{Q}_{C^{(r-1)}}(\chi)\right)\left(D_{r-1}\right)\right)\right. \\
& \approx H^{1}\left(D_{r-1}, \pi_{C}^{*}\left(\left.\left(\theta_{C} \otimes \mathscr{O}_{C}(\chi)\right)^{\otimes n+1}\left(D_{r-1}\right)\right|_{D_{r-1}} \otimes \tau_{r-1}^{*} \mathscr{O}_{C^{(r-1)}}(\chi)\right)\right.
\end{aligned}
$$

because

$$
H^{i}\left(C \times C^{(r-1)}, \pi_{C}^{*}\left(\left(\theta_{C} \otimes \mathscr{O}_{C}(\chi)\right)^{\otimes n+1} \otimes \pi_{C^{(r-1)}}^{*} \mathscr{O}_{C^{(r-1)}}(\chi)\right.\right.
$$

is zero for $i \leq 2$. This vanishing follows from the Künneth formula from $H^{0}\left(C,\left(\theta_{C} \otimes \mathscr{\mathscr { O }}_{C}(\chi)\right)\right)^{\otimes n+1}=0$ as the degree of the sheaf $<0$ for $g>2$ and $H^{i}\left(C^{(r-1)}, \mathscr{O}_{C^{(r-1)}}(\chi)\right)=0$ for $0 \leq i \leq 1$ by Corollary 11 .

Using the claim inductively we have an isomorphism $H^{1}\left(C^{(r)}, \theta_{C^{(r)}} \otimes \mathscr{O}_{C^{(r)}}(\chi)\right)$ $\stackrel{\approx}{\rightarrow} H^{1}\left(D_{2}, \pi_{C}^{*}\left(\left.\left(\theta_{C} \otimes \mathscr{O}_{C}(\chi)\right)^{\otimes(r-2)}\left(D_{2}\right)\right|_{D} \otimes \tau_{2}^{*} \mathscr{O}_{C(2)}(\chi)\right)\right.$ which by the first step of the claim is isomorphic to $H^{1}\left(C \times C,\left(\pi_{1}^{*}\left(\left(\theta_{C} \otimes \mathscr{O}_{C}(\chi)\right)^{\otimes r-1}\right) \otimes \pi_{2}^{*} \mathscr{O}_{C}(\chi)\right)(\Delta)\right)$. By duality on the surface $C \times C$ this group is dual to the cokernel of multiplication $m_{r}(\chi): \Gamma\left(C, \Omega_{C}^{\otimes r} \otimes \mathscr{O}_{C}\left(\chi^{-r+1}\right)\right) \otimes \Gamma\left(C, \Omega_{C} \otimes \mathscr{O}_{C}\left(\chi^{-1}\right)\right) \rightarrow \Gamma\left(C, \Omega_{C}^{\otimes r+1} \otimes \mathscr{O}_{C}\left(\chi^{-r}\right)\right)$. Thus we need to have $m_{r}(\chi)$ surjective. 
We first need to have $\Gamma\left(C, \Omega_{C} \otimes \mathscr{O}_{C}\left(\chi^{-1}\right)\right)$ has no base points; i.e. $\mathscr{O}_{C}\left(\chi^{-1}\right) \not z$ $\mathscr{O}_{C}\left(c_{1}-c_{2}\right)$ for two points $c_{1}$ and $c_{2}$ on $C$. Otherwise $\mathscr{O}_{C}=\left(\mathscr{O}_{C}(\chi)\right)^{\operatorname{deg} f}=$ $\mathscr{O}_{C}\left(\operatorname{deg} f \cdot c_{1}-\operatorname{deg} f \cdot c_{2}\right)$. Therefore there is a morphism $G: C \rightarrow \mathbb{P}^{n}$ of $\operatorname{deg}=\operatorname{deg} f$ with only one point over both 0 and $\infty$. By Riemann-Hurwitz formula $G$ has $2 g$ other ramification points. Normalizing one to be over $1, G$ depends on $2 g-1$ parameters, but $2 g-1<3 g-3=\operatorname{dim}$ (Moduli) as $g>2$. Thus we have no base points for a general curve. By $r>3\left(\Omega_{C}^{\otimes r} \otimes \mathscr{O}_{C}\left(\chi^{-r+1}\right)\right) \otimes$ $\left(\Omega_{C} \otimes \mathscr{O}_{C}\left(\chi^{-1}\right)\right)$ is not special $(g>1)$. Thus for general $C$ the surjectivity of $m_{r}(\chi)$ follows from the original Castelnuovo lemma.

Remark. X. Gomezmont has extended the results of [2] to all curves of genus $>2$.

\section{REFERENCES}

1. G. Kempf, Toward the inversion of abelian integrals, II, Amer. Jour. of Math. 101 (1979) 184-202.

2. __ Deformations of symmetric products, in "Riemann Surfaces and Related Topics", Proceeding of the 1978 Stony Brook Conference, Princeton University Press, Princeton, 1980.

3. _- Multiplication over abelian varieties, (to appear).

4. __ Notes on the inversion of integrals I, Proc. Amer. Math. Soc., (to appear).

5. S. Koizumi, Theta relations and projective normality of abelian varieties, Amer. Jour. of Math. 98 (1976) 865-889.

6. _ The rank theorem on matrices of theta functions, J. Fac. of Sci., University of Tokyo, 24 (1977) 115-122.

7. D. Mumford, On the equations defining abelian varieties, I, Inv. Math. 1 (1966), 287-354.

8. _ Varieties defined by quadratic equations, in "Questions on Algebraic Varieties", Centro Inter. Mate. Estrivo, Roma, 1970, 31-100.

Department of Mathematics, The Johns Hopkins University, Baltimore, Maryland 21218 